\title{
Conditioning factors determination for landslide susceptibility mapping using support vector machine learning
}

\begin{abstract}
This study investigates the effectiveness of two sets of landslide conditioning variable(s). Fourteen landslide conditioning variables were considered in this study where they were duly divided into two sets G1 and G2. Two Support Vector Machine (SVM) classifiers were constructed based on each dataset (SVM-G1 and SVM-G2) in order to determine which set would be more suitable for landslide susceptibility prediction. In total, 160 landslide inventory datasets of the study area were used where $70 \%$ was used for SVM training and $30 \%$ for testing. The intra-relationships between parameters were explored based on variance inflation factors (VIF), Pearson's correlation and Cohen's kappa analysis. Other evaluation metrics are the area under curve (AUC).
\end{abstract}

Keyword: Support vector machine; Conditioning factors; Factor correlation; Iran 